\title{
The Incomplete Beta Function and its Ratio to the Complete Beta Function
}

\section{By David Osborn and Richard Madey*}

1.Abstract. The incomplete beta function, $B_{x}(p, q)=\int_{0}^{x} y^{p-1}(1-y)^{q-1} d y$, and its ratio to the complete beta function $B_{1}(p, q)$ has been calculated on an IBM-7044 computer to five significant figures and tabulated for the arguments $p$ and $q$ each in the interval from 0.5 to 2.0 with increments of 0.05 and for the parameter $x$ in the interval 0.1 to 1.0 with increments of 0.01 . The function was evaluated by first expanding a factor of the integrand in a binomial series and then integrating each term. In order to facilitate computation, one expansion is taken for $x \leqq 1 / 2$ and another for $1 / 2<x \leqq 1$. The expansions are truncated when the relative error is less than or equal to an arbitrarily predetermined fraction. Typical running time for achieving five significant figures on the IBM-7044 is about one minute for $10^{4}$ values.

2. Introduction. In a paper published by Madey and Stephenson [1] and in the authors' present research on the quality factor (QF) for degraded heavy particle spectra observed in solar flare activity, the QF is expressed in terms of complete and incomplete beta functions. The incomplete beta function is defined by the integral [2], [3]

$$
B_{x}(p, q)=\int_{0}^{x} y^{p-1}(1-y)^{q-1} d y \quad(0<x \leqq 1) .
$$

The parameter $p$ is restricted to positive values in order to avoid the singularity at $y=0$. There is no restriction on $q$ if $x<1$; however, when the upper limit $x=1$, $q$ must be greater than zero in order to avoid the singularity at $y=1$. When the upper limit $x$ is equal to unity, the integral becomes the beta function, $B(p, q)$. It is worth noting that $B(p, q)=B(q, p)$ but that $B_{x}(p, q) \neq B_{x}(q, p)$. The observed spectral exponents in power-law representations of the incident differential flux spectra are directly related to the arguments $p$ and $q$ of the incomplete beta function. The values of the incomplete beta function for the arguments $p$ and $q$ in the interval from about 0.5 to 2.0 are needed to determine the QF. A table of the incomplete beta function in this range does not appear to exist in the open literature [4], [11]-[20] for small enough increments of the arguments.

The incomplete beta quotient is related to the cumulative binomial distribution by the relation $[3],[6]$ :

$$
I_{x}(p, n-p+1) \equiv \frac{B_{x}(p, n-p+1)}{B(p, n-p+1)}=\sum_{s=p}^{n}\left(\begin{array}{c}
n \\
s
\end{array}\right) x^{s}(1-x)^{n-s} .
$$

Here, the parameter $q$ in Eq. (1) is related to the parameter $p$ through the relation

Received March 1, 1967. Revised May 8, 1967.

* Address communications regarding this paper to Richard Madey. 
$q=n-p+1$. Also, since $s$ must be an integer, $p$ and $q$ are restricted to integers. Tables of the cumulative binomial distribution have been published by the National Bureau of Standards [5], Simon \& Grubbs [6], Romig [7], Harvard University Computational Laboratory [8], and Weintraub [9].

The complete beta function may also be obtained from the gamma function through the following well-known relation [2], [3].

$$
B(p, q)=\frac{\Gamma(p) \Gamma(q)}{\Gamma(p+q)} .
$$

Davis [3] tabulates the gamma function to $10 \mathrm{D}$ for values of the argument in the interval from 1.000 to 2.000 at increments of .005 and to $11 \mathrm{~S}$ for integer and halfinteger values of the argument in the interval from 1 to 101 .

3. Analysis. The incomplete beta function can be evaluated by first taking a binomial expansion of the factor $(1-y)^{q-1}$ in the integrand and then integrating term by term:

$$
\begin{aligned}
B_{x}(p, q)=x^{p}\left[\frac{1}{p}+\frac{1-q}{p+1}\right. & x+\frac{(1-q)(2-q)}{2 !(p+2)} x^{2} \\
& \left.+\frac{(1-q)(2-q)(3-q)}{3 !(p+3)} x^{3}+\cdots\right],
\end{aligned}
$$

where $p \neq 0,-1,-2, \cdots$. The error in truncating the series of Eq. (4) is determined by the well-known theorem [10] which states that the remainder after $k$ terms of a series of positive terms is less than $r /(1-r)$ times the last term retained, if $u_{i+1} / u_{i} \leqq r$ for $i \geqq k$. The criterion for truncating the series is that the relative error be less than $10^{-m}$, where $m$ denotes the number of significant figures desired. The relative error is taken to be the ratio $R_{k}$, the remainder after $k$ terms, to $S_{k}$, the sum of the first $k$ terms.

The ratio of the $(k+1)$ th term to the $k$ th term of Eq. (4) is

$$
\frac{U_{k+1}}{U_{k}}=\frac{(p+k)(k+1-q)}{(p+k+1)(k+1)} x
$$

and

$$
\operatorname{Lim}_{k \rightarrow \infty} \frac{U_{k+1}}{U_{k}}=x .
$$

According to this ratio test, the series in Eq. (4) converges for $|x|<1$. Since the preceding error theorem cannot be applied for $x=1$, another approach is needed to determine the complete beta function in this case. The integral of Eq. (2) can be transformed as follows:

$$
B_{x}(p, q)=B_{1 / 2}(p, q)+\int_{1 / 2}^{x} y^{p-1}(1-y)^{q-1} d y
$$

which, with the substitution $y=1-z$, becomes

$$
B_{x}(p, q)=B_{1 / 2}(p, q)+\int_{1-x}^{1 / 2} z^{q-1}(1-z)^{p-1} d z .
$$


As before, the integral in Eq. (7) is evaluated by first taking a binomial expansion of the factor $(1-z)^{p-1}$ in the integrand and then integrating term by term. Thus,

$$
\begin{aligned}
B_{x}(p, q)= & B_{1 / 2}(p, q)+\frac{\left(1-w^{q}\right)}{q 2^{q}}+\frac{(1-p)\left(1-w^{q+1}\right)}{1 !(q+1) 2^{q+1}} \\
& +\frac{(1-p)(2-p)\left(1-w^{q+2}\right)}{2 !(q+2) 2^{q+2}}+\cdots
\end{aligned}
$$

where $w=2(1-x)$ and $q \neq 0,-1,-2, \cdots$. The ratio of the $(k+1)$ th term to the $k$ th term of Eq. (8) is

$$
\frac{U_{k+1}}{U_{k}}=\frac{(q+k)(k+1-p)}{(q+k+1)(k+1)}(x-1 / 2)
$$

and

$$
\operatorname{Lim}_{k \rightarrow \infty} \frac{U_{k+1}}{U_{k}}=x-1 / 2 .
$$

According to this ratio test, the series in Eq. (8) converges for $|x-1 / 2|<1$, that is, for $-1 / 2<x<3 / 2$. Comparison of Eqs. (5) and (9) indicates that the two series in Eqs. (4) and (8) will converge with approximately the same number of terms, provided that the series of Eq. (4) is used when $0<x \leqq 1 / 2$ and that the series of Eq. (8) is used when $1 / 2<x \leqq 1$. The fact that the series of Eq. (4) cannot be used when $x=1$, and the fact that convergence is more rapid for the above two series in Eqs. (4) and (8) than for the one series alone in Eq. (4), provides the basis for choosing the two series.

4. Computer Program. This program calculates the incomplete beta function $B_{x}(p, q)$ and its ratio to the complete beta function $B(p, q)$ for allowable ranges of the parameters $p$ and $q$. Excluded are values of $p<0$ for all allowed values of $x$ and values of $q<0$ only if $x=1$. By definition, the parameter $x$ varies between 0 and 1 . The ranges and increments of the arguments $p$ and $q$ and the parameter $x$, and the number of significant figures desired are input data. This program was originally written in Forgo language for the IBM-1620 computer at Clarkson College of Technology. It was also written in Fortran II and Fortran IV and run on the IBM-7044 computer at the University of Buffalo.

The integral defining the incomplete beta function is evaluated after first expanding one factor in the integrand by the binomial theorem. In order to obtain more rapid convergence, one expansion is taken for $x \leqq 1 / 2$ and another for $1 / 2<x \leqq 1$. This method of solution imposes the further restriction that $q \neq$ $0,-1,-2, \cdots$. The expansions are truncated when the relative error is less than or equal to an arbitrarily predetermined fraction. Several values are calculated and stored in memory and then are punched out in a page format. Typical running time for achieving five significant figures on the IBM-1620 is about one minute per 2.7 values while that on the IBM-7044 is less than one minute per $10^{4}$ values.

5. Results. Values of the incomplete beta function, the complete beta function, and the ratio of the incomplete beta function to the complete beta function have 
thus been calculated to $5 \mathrm{D}$ for $p, q=0.5(0.05) 2.05, \quad x=0.1(0.01) 1$ [21]. An abridgement corresponding to $p, q=0.5(0.1) 2, x=0.1(0.1) 1$ is given here in microfiche. We have checked our computations with those of Pearson [4] for comparable ranges of the arguments. Pearson has calculated these functions only for values of $p$ and $q$ equal to half-integers and for values of $p$ which are greater than $q$. To compare those values for which $p$ is greater than $q$, we made use of the formula $I_{1-x}(p, q)=1-I_{x}(q, p)$. All of our values for $p$ and $q$ equal to half-integers agreed with those of Pearson to within one part in $10^{5}$.

Acknowledgment. The authors wish to acknowledge communication with Dr. John W. Wrench, Jr. of the David Taylor Model Basin relating to tables of the incomplete beta function. The authors are appreciative of the opportunity to use the IBM-7044 computer at the University of Buffalo.

Department of Physics

Clarkson College of Technology

Potsdam, New York 13676

1. Richard Madey \& T. E. StePhenson, "Quality factors for degraded proton spectra," Proceedings of the Second Symposium on Protection Against Radiations in Space (held at Gatlinburg, Tennessee, October 1964), NASA SP-71, 229, 1965.

2. E. T. WhitTaker \& G. M. Watson, A Course of Modern Analysis, 4th ed., Cambridge Univ. Press, New York, 1927; 1946, pp. 253-254.

3. P. J. DAvis, "Gamma function and related functions," Ch. 6 in Handbook of Mathematical Functions with Formulas, Graphs, and Mathematical Tables, edited by Milton Abramowitz and I. A. Stegun, National Bureau of Standards, Applied Math. Series, No. 55, U. S. Government Printing Office, Washington, D. C., 1964, MR 29 \#4914.

4. Kard Pearson, Tables of the Incomplete Beta-Function, Cambridge Univ. Press, New York, 1934.

5. Tables of the Binomial Probability Distribution, National Bureau of Standards, Applied Math. Series, No. 6, U. S. Government Printing Office, Washington, D. C., 1950.

6. L. E. Simon \& F. E. GrubBs, Tables of the Cumulative Binomial Probabilities, Ballistic Research Laboratories Ordinance Corps. Pamphlet ORDP20-1, Aberdeen Proving Ground, Maryland, 1952 and 1956 Supplement.

7. H. G. Romig, 50-100 Binomial Tables, Wiley, New York; Chapman \& Hall, London, 1953. MR 15, 63.

8. Tables of the Cumulative Binomial Probability Distribution, Annals of the Harvard University Computation Laboratory, Vol. 35, Harvard Univ. Press, Cambridge, Mass., 1955. MR 18,517 .

9. Sol Weintraub, Tables of the Cumulative Binomial Probability Distribution for Small Values of $p$, The Free Press of Glencoe, New York; Collier-Macmillan, London, 1963. MR 28 $\# 1672$.

10. R. R. Middlemiss, Differential and Integral Calculus, McGraw-Hill, New York, 1940; 1946, p. 407. MR 1, 299.

11. R. S. BuRINGTON \& D. C. MAY, Handbook of Probability and Statistics with Tables, Handbook Publishers, Sandusky, Ohio, 1953.

12. A. Erdélyi, W. Magnus, F. Oberhettinger \& F. Tricomi, Higher Transcendental Functions, Vol. 1, Bateman Manuscript Project, McGraw-Hill, New York, 1953. MR 15, 419.

13. A. Fletcher, J. C. P. Miller \& L. Rosenhead, Index of Mathematical Tables, McGrawHill, New York, 1946. MR 8, 286.

14. J. A. Greenwood \& H. O. Harteey, Guide to Tables in Mathematical Statistics, Princeton Univ. Press, Princeton, N. J., 1962, pp. 181-206. MR 27 \#4299.

15. Karl Pearson, Tables for Statisticians and Biometricians, Cambridge Univ. Press, New York, 1914 .

16. Karl Pearson, Tables for Statisticians and Biometricians, Part II, London, 1935, p. 240.

17. Karl Pearson \& Margaret Pearson, "On the numerical evaluation of high order incomplete Eulerian integrals," Biometrika, v. 27, 1935, pp. 409-423.

18. Reference Handbook of the Boulder Laboratories Library. Part III: Mathematical Tables, 2nd ed., U. S. Dept. of Commerce, National Bureau of Standards, Boulder, Colorado, 1964.

19. H. E. Soper, The Numerical Evaluation of the Incomplete B-Function, Cambridge Tracts for Computers, No. 7, Cambridge Univ. Press, New York, 1921.

20. J. WISHART, "On the approximate quadrature of certain skew curves, with an account of the researches of Thomas Bayes," Biometrika, v. 19, 1927, pp. 1-38.

21. Richard MadeY \& David Osborn, Tables of the Incomplete Beta Function and Its Ratio to the Complete Beta Function. (Unpublished work copyrighted 1967.) 